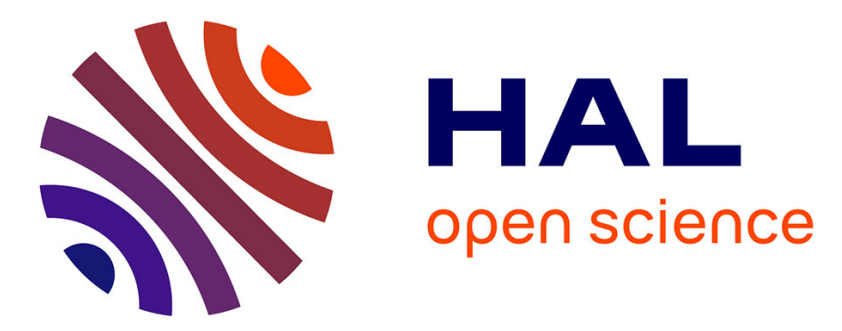

\title{
Asymptotic-Preserving scheme based on a Finite Volume/Particle-In-Cell coupling for Boltzmann- BGK-like equations in the diffusion scaling
}

\author{
Anaïs Crestetto, Nicolas Crouseilles, Mohammed Lemou
}

\section{- To cite this version:}

Anaïs Crestetto, Nicolas Crouseilles, Mohammed Lemou. Asymptotic-Preserving scheme based on a Finite Volume/Particle-In-Cell coupling for Boltzmann- BGK-like equations in the diffusion scaling. Finite Volumes for Complex Applications VII, Jun 2014, Berlin, Germany. p. 827, 10.1007/978-3319-05591-6_83 . hal-01015936

\section{HAL Id: hal-01015936 \\ https://hal.science/hal-01015936}

Submitted on 27 Jun 2014

HAL is a multi-disciplinary open access archive for the deposit and dissemination of scientific research documents, whether they are published or not. The documents may come from teaching and research institutions in France or abroad, or from public or private research centers.
L'archive ouverte pluridisciplinaire HAL, est destinée au dépôt et à la diffusion de documents scientifiques de niveau recherche, publiés ou non, émanant des établissements d'enseignement et de recherche français ou étrangers, des laboratoires publics ou privés. 


\title{
Asymptotic-Preserving scheme based on a Finite Volume/Particle-In-Cell coupling for Boltzmann- BGK-like equations in the diffusion scaling
}

\author{
Anaïs Crestetto, Nicolas Crouseilles and Mohammed Lemou
}

\begin{abstract}
This work is devoted to the numerical simulation of the collisional Vlasov equation in the diffusion limit using particles. To that purpose, we extend the Finite Volumes/Particles hybrid scheme developed in [5], based on a micro-macro decomposition technique introduced in [1] or [13]. Whereas a uniform grid was used to approximate both the micro and the macro part of the full distribution function in [13], we use here a particle approximation for the kinetic (micro) part, the fluid (macro) part being always discretized by standard finite volume schemes. There are many advantages in doing so: (i) the so-obtained scheme presents a much less level of noise compared to the standard particle method; (ii) the computational cost of the micro-macro model is reduced in the diffusion limit since a small number of particles is needed for the micro part; (iii) the scheme is asymptotic preserving in the sense that it is consistent with the kinetic equation in the rarefied regime and it degenerates into a uniformly (with respect to the Knudsen number) consistent (and deterministic) approximation of the limiting equation in the diffusion regime.
\end{abstract}

Anaïs Crestetto

Université de Nantes, Laboratoire de Mathématiques Jean Leray, 2 rue de la Houssinière 44322

Nantes France, e-mail: anais.crestetto@univ-nantes.fr

Nicolas Crouseilles

INRIA Rennes Bretagne-Atlantique, projet IPSO \& Université de Rennes 1, Institut de Recherche Mathématique de Rennes, 263 avenue du Général Leclerc 35042 Rennes France, e-mail: nicolas.crouseilles@inria.fr

Mohammed Lemou

CNRS \& Université de Rennes 1, Institut de Recherche Mathématique de Rennes, 263 avenue du Général Leclerc 35042 Rennes France, e-mail: mohammed.lemou@univ-rennes1.fr 


\section{Introduction}

Particle systems appearing in several physical applications like plasma or radiative transfer can be studied at different scales. A kinetic description is necessary when the system is far from thermodynamical equilibrium. It is based on the representation of the set of particles by a distribution function $f$ depending on time $t$, space $x$ and velocity $v, f$ verifying a partial differential equation of Vlasov-type. When the system stays near equilibrium, the problem can be reduced using a macroscopic description, only depending on $t$ and $x$. Several strategies can be used to solved multiscale problems (see for example [9], [8], [11] or [2]), among them, the micro-macro decomposition introduced in [1] leads to a coupling of two equations: a macroscopic one for the mean part of $f$ (in velocity) and a microscopic one for the remainder part (called perturbation).

This work is devoted to the design of an Asymptotic-Preserving (AP) scheme (see [10]) for the following kinetic equation in the diffusion scaling

$$
\partial_{t} f+\frac{1}{\varepsilon} v \partial_{x} f+\frac{1}{\varepsilon} E \partial_{v} f=\frac{1}{\varepsilon^{2}}(\rho M-f)
$$

where $x \in\left[0, L_{x}\right], \rho=\int f d v$ is the charge density, $E$ is the electric field given by the Poisson equation $\partial_{x} E=\rho-1, M$ is either the absolute Maxwellian (in the BGKcase $v \in \mathbb{R}$ ) or equal to 1 (in the radiative transport equation (RTE)-case $v \in[-1,1]$ ) and $\varepsilon$ is the Knudsen number, parameter of the frequency of collisions between particles, that may be of order one or tend to zero in the diffusion limit.

The strategy will be the use of the micro-macro decomposition. However, following [5], we want to use particles to discretize the micro part so that in the limit regime, the numerical cost is reduced since a few number of particles will be necessary to sample the (small) non equilibrium part. The main difficulty compared to phase space grid approaches $[13,6]$ remains in the fact that the use of particles requires a splitting between transport and source terms whereas in $[13,6]$, the stiffest (source) term is used to stabilize the stiff transport term.

The outline of the paper is the following. We derive the micro-macro model in Section 2 and its numerical discretization in Section 3. Some numerical results are given in Section 4. Section 5 is devoted to the conclusion and some perspectives.

\section{Derivation of the micro-macro equations}

This section is devoted to the derivation of the micro-macro model. Let us first introduce a velocity-set $V=\mathbb{R}$ in the Vlasov-Poisson-BGK-case and $V=[-1,1]$ in the RTE-case and define the null space of the linear collisional BGK-operator $Q(f)=\rho M-f$ by $\mathscr{N}=\operatorname{Span}\{M\}=\{f=\rho M$, with $\rho=\langle f\rangle\}$, where $\langle h\rangle:=\int h d v$ and $M(v)=\frac{1}{\sqrt{2 \pi}} \exp \left(-\frac{v^{2}}{2}\right)$ is the absolute Maxwellian in the Vlasov-Poisson-case or $M=1$ in the RTE-case. We now define the orthogonal projector $\Pi$ in $L^{2}\left(M^{-1} \mathrm{~d} v\right)$ 
onto $\mathscr{N}$ as $\Pi h:=\langle h\rangle M$. Following [13,6], we decompose $f$ as $f=\rho M+g$, where $\rho:=\langle f\rangle$ and $g:=f-\rho M$, and rewrite the kinetic equation (1) into the equivalent micro-macro model

$$
\left\{\begin{array}{l}
\partial_{t} \rho+\frac{1}{\varepsilon} \partial_{x}\langle v g\rangle=0 \\
\partial_{t} g+\frac{1}{\varepsilon}(I-\Pi)\left[v \partial_{x}(\rho M+g)+E \partial_{v}(\rho M+g)\right]=-\frac{1}{\varepsilon^{2}} g
\end{array}\right.
$$

The micro equation on $g$ makes appear stiff terms that need a particular treatment in order to get an AP scheme. The strategy of [12] is used and recalled here. We rewrite the flux term of the micro equation

$$
\begin{aligned}
(I-\Pi)\left(v \partial_{x}(\rho M+g)\right) & =v M \partial_{x} \rho+v \partial_{x} g-\partial_{x} \Pi(v g), \\
(I-\Pi)\left(E \partial_{v}(\rho M+g)\right) & =E \partial_{v}(\rho M+g)=-v M E \rho+E \partial_{v} g
\end{aligned}
$$

so that the micro equation becomes

$$
\partial_{t} g+\frac{1}{\varepsilon}\left[v M \partial_{x} \rho+v \partial_{x} g-\partial_{x}\langle v g\rangle M-v M E \rho+E \partial_{v} g\right]=-\frac{1}{\varepsilon^{2}} g .
$$

Starting from (3), we rewrite it as

$$
\partial_{t}\left(e^{t / \varepsilon^{2}} g\right)=-\frac{e^{t / \varepsilon^{2}}}{\varepsilon}\left[v M \partial_{x} \rho+v \partial_{x} g-\partial_{x}\left\langle v g^{n}\right\rangle M-v M E \rho+E \partial_{v} g\right]
$$

Integrating in time between $t^{n}$ and $t^{n+1}$ leads to

$e^{t^{n+1} / \varepsilon^{2}} g^{n+1}=e^{t^{n} / \varepsilon^{2}} g^{n}-\frac{1}{\varepsilon} \int_{t^{n}}^{t^{n+1}} e^{t / \varepsilon^{2}} d t\left[v M \partial_{x} \rho^{n}+v \partial_{x} g^{n}-\partial_{x}\left\langle v g^{n}\right\rangle M-v M E^{n} \rho^{n}+E^{n} \partial_{v} g^{n}\right]$,

and multiplying by $e^{-t^{n+1} / \varepsilon^{2}}$ gives

$g^{n+1}=e^{-\Delta t / \varepsilon^{2}} g^{n}-\varepsilon\left(1-e^{-\Delta t / \varepsilon^{2}}\right)\left[v M \partial_{x} \rho^{n}+v \partial_{x} g^{n}-\partial_{x}\left\langle v g^{n}\right\rangle M-v M E^{n} \rho^{n}+E^{n} \partial_{v} g^{n}\right]$.

By using the discrete time derivative, we finally get

$$
\begin{array}{r}
\frac{g^{n+1}-g^{n}}{\Delta t}=\frac{\left(e^{-\Delta t / \varepsilon^{2}}-1\right)}{\Delta t} g^{n}-\varepsilon \frac{\left(1-e^{-\Delta t / \varepsilon^{2}}\right)}{\Delta t}\left[v M \partial_{x} \rho^{n}+v \partial_{x} g^{n}\right. \\
\left.-\partial_{x} \Pi\left(v g^{n}\right) M-v M E^{n} \rho^{n}+E^{n} \partial_{v} g^{n}\right],
\end{array}
$$

which we approximate, up to terms of order $\mathscr{O}\left(\Delta t^{2}\right)$, by

$$
\partial_{t} g=\frac{e^{-\Delta t / \varepsilon^{2}}-1}{\Delta t} g-\varepsilon \frac{1-e^{-\Delta t / \varepsilon^{2}}}{\Delta t}\left[v M \partial_{x} \rho+v \partial_{x} g-\partial_{x} \Pi(v g) M-v M E \rho+E \partial_{v} g\right]
$$

Let us remark that this equation does not contain any stiff term. Moreover, the two following properties are verified:

- consistency: $\forall \varepsilon>0$ fixed, as $\Delta t \rightarrow 0$, we recover the initial micro equation (3), 
- AP property: $\forall \varepsilon>0$ fixed, as $\varepsilon \rightarrow 0$, we get $g=-\varepsilon\left(v M \partial_{x} \rho-v M E \rho\right)$, which injected in the macro equation provides the right limit model given by $\partial_{t} \rho-$ $\partial_{x}\left(\partial_{x} \rho-E \rho\right)=0($ see $[6])$.

The next section is devoted to the numerical discretization of the modified micromacro model

$$
\begin{aligned}
& \partial_{t} \rho+\frac{1}{\varepsilon} \partial_{x}\langle v g\rangle=0, \\
& \partial_{t} g=\frac{e^{-\Delta t / \varepsilon^{2}}-1}{\Delta t} g-\varepsilon \frac{1-e^{-\Delta t / \varepsilon^{2}}}{\Delta t}\left[v M \partial_{x} \rho+v \partial_{x} g-\partial_{x} \Pi(v g) M-v M E \rho+E \partial_{\nu} g\right]
\end{aligned}
$$

by an hybrid scheme, that couples finite volumes for the macro part $\rho$ to a particle method for the micro part $g$. As in [5], where the hydrodynamic limit was studied, we expect a reduction of computational time when $\varepsilon \rightarrow 0$, related to the few number of particles needed to represent $g$ at the limit.

\section{Finite Volumes/Particles discretization}

We present in this section the Finite Volumes/Particle-In-Cell (PIC) coupling developed for solving (5). Such a coupling is explained in more details in [5] for the hydrodynamic limit.

Let us consider a classical uniform discretization of the spatial domain $x \in\left[0, L_{x}\right]$ denoted by $\left(\mathrm{x}_{i}\right)_{0 \leq i \leq N_{x}}$ and the following approximations: $\rho_{i}^{n} \approx \rho\left(t^{n}, \mathrm{x}_{i}\right)$ and $E_{i}^{n} \approx$ $E\left(t^{n}, \mathrm{x}_{i}\right)$. The Poisson equation $\partial_{x} E=\rho-1$ for $E$ is solved thanks to finite volumes without difficulty. We now focus on the two other equations (5).

\subsection{Particle approximation for $g$}

Our goal is to extend the particle discretization developed in [5] to the diffusion scaling. To that purpose, we exploit the reformulation (4). As already said in [5], we have to use a splitting procedure between the transport part and the source part. Then, the algorithm is the following

- solve $\partial_{t} g+\varepsilon \frac{\left(1-e^{\left.-\Delta t / \varepsilon^{2}\right)}\right.}{\Delta t} v \partial_{x} g+\varepsilon \frac{\left(1-e^{-\Delta t / \varepsilon^{2}}\right)}{\Delta t} E \partial_{v} g=0$,

- solve $\partial_{t} g=\frac{\left(e^{-\Delta t / \varepsilon^{2}}-1\right)}{\Delta t} g-\varepsilon \frac{\left(1-e^{-\Delta t} / \varepsilon^{2}\right)}{\Delta t}\left[v M \partial_{x} \rho+\partial_{x}\langle v g\rangle M-v M E \rho\right]$.

In the PIC method (described for example in [3]), the distribution function $g$ is represented by a set of $N$ particles of position $x_{k}$, velocity $v_{k}$ and weight $\omega_{k}$ and approximated by $g(t, x, v)=\sum_{k=1}^{N} \omega_{k}(t) \delta\left(x-x_{k}(t)\right) \delta\left(v-v_{k}(t)\right)$. Then, the transport part is solved with the (non stiff) characteristics

$$
\dot{x}_{k}=\varepsilon \frac{\left(1-e^{-\Delta t / \varepsilon^{2}}\right)}{\Delta t} v_{k}, \quad \dot{v}_{k}=\varepsilon \frac{\left(1-e^{-\Delta t / \varepsilon^{2}}\right)}{\Delta t} E\left(t, x_{k}\right),
$$


$E\left(t, x_{k}\right)$ being computed by a deposition step knowing $E_{i}^{n}$ on the mesh. The source part is solved using the equation satisfied by the weights

$$
\begin{array}{r}
\dot{\omega}_{k}=\frac{\left(e^{-\Delta t / \varepsilon^{2}}-1\right)}{\Delta t} \omega_{k}-\varepsilon \frac{\left(1-e^{-\Delta t / \varepsilon^{2}}\right)}{\Delta t}\left[v _ { k } M ( v _ { k } ) \left(\partial_{x} \rho\left(t, x_{k}\right)\right.\right. \\
\left.\left.-E\left(t, x_{k}\right) \rho\left(t, x_{k}\right)\right)+\partial_{x}\langle v g\rangle\left(t, x_{k}\right) M\left(v_{k}\right)\right] .
\end{array}
$$

In more details, from an initial repartition of the $N$ particles $\left(x_{k}^{0}, v_{k}^{0}\right)$ in the phasespace domain of size $L_{x} \times L_{v}$, with $\omega_{k}^{0}=g\left(t=0, x_{k}, v_{k}\right) L_{x} L_{v} / N$, (6) is approximated by

$$
x_{k}^{n+1}=x_{k}^{n}+\varepsilon\left(1-e^{-\Delta t / \varepsilon^{2}}\right) v_{k}^{n}, v_{k}^{n+1}=v_{k}^{n}+\varepsilon\left(1-e^{-\Delta t / \varepsilon^{2}}\right) E^{n}\left(x_{k}^{n}\right) .
$$

Then, we compute the momentum $\left\langle v g^{n+1 / 2}\right\rangle$ of $g^{n+1 / 2}$ using this new position:

$$
\left.\left\langle v g^{n+1 / 2}\right\rangle\right|_{x=\mathrm{X}_{i}} \approx \sum_{k=1}^{N} \omega_{k}^{n} B_{\ell}\left(\mathrm{x}_{i}-x_{k}^{n+1}\right) v_{k}^{n+1}
$$

$B_{\ell} \geq 0$ is a B-spline function of order $\ell$ :

$$
B_{\ell}(x)=\left(B_{0} * B_{\ell-1}\right)(x), \quad \text { with } B_{0}(x)= \begin{cases}\frac{1}{\Delta x} & \text { if }|x|<\Delta x / 2 \\ 0 & \text { else. }\end{cases}
$$

We rewrite the weight equation as

$$
\omega_{k}^{n+1}=\omega_{k}^{n}+\left(e^{-\Delta t / \varepsilon^{2}}-1\right) \omega_{k}^{n}-\varepsilon\left(1-e^{-\Delta t / \varepsilon^{2}}\right)\left[\alpha_{k}^{n}+\beta_{k}^{n}\right],
$$

with $\alpha_{k}^{n}=v_{k} M\left(v_{k}\right)\left[\partial_{x} \rho^{n}\left(x_{k}\right)-E^{n}\left(x_{k}\right) \rho^{n}\left(x_{k}\right)\right] \frac{L_{x} L_{v}}{N}$ and $\beta_{k}^{n}=\partial_{x}\left\langle v g^{n}\right\rangle\left(x_{k}\right) M\left(v_{k}\right) \frac{L_{x} L_{v}}{N}$. To compute $\alpha_{k}^{n}$ (resp. $\beta_{k}^{n}$ ), since $\rho^{n}$ (resp. $\left\langle v g^{n}\right\rangle$ ) is known on the spatial grid, we approximate $\partial_{x} \rho^{n}$ (resp. $\left.\partial_{x}\left\langle v g^{n}\right\rangle\right)$ by centered finite differences: $\left(\partial_{x} \rho^{n}\right)_{i} \approx \frac{\rho_{i+1}^{n}-\rho_{i-1}^{n}}{2 \Delta x}$ (resp. $\left(\partial_{x}\left\langle v g^{n}\right\rangle\right)_{i} \approx \frac{\left\langle v g^{n}\right\rangle_{i+1}-\left\langle v g^{n}\right\rangle_{i-1}}{2 \Delta x}$ ) and evaluate at $x=x_{k}$ using an interpolation.

Remark. We have now a new approximation of $g^{n+1}$ given by its particle discretization. We have to ensure that the micro-macro structure $f=\rho M+g$ with $\rho=\int f d v$ is preserved numerically. To do that, we correct the weights $\omega_{k}^{n+1}$, adapting an idea of [7]. We do not detail this procedure here but refer the reader to [5].

Chapman-Enskog expansion. When $\varepsilon$ goes to zero, we immediatly observe that $\omega_{k}^{n+1}=-\varepsilon \alpha_{k}^{n}+\mathscr{O}\left(\varepsilon^{2}\right)$ (since $\omega_{k}^{n}=\mathscr{O}(\varepsilon) \forall n \geq 1$ ). Computing the momentum of $g^{n+1}$ means that we use (9) with $g^{n+1}$, or in the limit regime

$$
\begin{aligned}
\left.\left\langle v g^{n+1}\right\rangle\right|_{x=\mathrm{x}_{i}} & \approx-\varepsilon \sum_{k=1}^{N} \alpha_{k}^{n} B_{\ell}\left(\mathrm{x}_{i}-x_{k}\right) v_{k}+\mathscr{O}\left(\varepsilon^{2}\right), \\
& \approx-\left.\varepsilon\left[\left\langle v^{2} M\right\rangle\left(\partial_{x} \rho^{n}-E^{n} \rho^{n}\right)\right]\right|_{x=\mathrm{x}_{i}}+\mathscr{O}\left(\varepsilon^{2}\right) \\
& \approx-\varepsilon\left(\partial_{x} \rho^{n}-E^{n} \rho^{n}\right)\left(\mathrm{x}_{i}\right)+\mathscr{O}\left(\varepsilon^{2}\right) .
\end{aligned}
$$

Injecting in the macro equation then leads to a discretization of $\partial_{t} \rho-\partial_{x}\left(\partial_{x} \rho-E \rho\right)=$ 0 , which corresponds to the right asymptotic model (see [6]). 


\subsection{Coupling strategy}

After the computation of $g^{n+1}$ by the PIC method, we compute $\rho^{n+1}$ thanks to a standard finite volume method. We use for example the following scheme:

$$
\rho_{i}^{n+1}=\rho_{i}^{n}-\frac{1}{\varepsilon} \frac{\Delta t}{\Delta x}\left(\left\langle v g^{n+1}\right\rangle_{i+\frac{1}{2}}-\left\langle v g^{n+1}\right\rangle_{i-\frac{1}{2}}\right)
$$

where $\left\langle v g^{n+1}\right\rangle_{i+\frac{1}{2}}$ is computed with (9).

Finally, the algorithm reduces to:

- Initialization of $\left(x_{k}, v_{k}\right)$ and $\omega_{k}$.

- 1) Advance micro part: - advance the characteristics with (8),

- compute $\left\langle v g^{n}\right\rangle$ with (9),

- advance the weights equation with (11).

- 2) Correction step for preserving the micro-macro structure as in [5].

- 3) Advance macro part: - compute $\left\langle v g^{n+1}\right\rangle$ with (9), - compute $\rho^{n+1}$ with (12).

\section{Numerical results}

We validate our model, denoted by MiMa-Part, on two classical test cases and compare it to a full particle method ( $f$ is discretized by particles and not only $g$, see [3]) denoted by Full-PIC and to a micro-macro scheme using a Eulerian discretization of phase space, denoted by MiMa-Grid (which corresponds to the scheme developed in [6]).

We first consider the linear Landau damping case, where $f$ is initially given by $f(0, x, v)=\frac{1}{\sqrt{2 \pi}} \exp \left(-\frac{v^{2}}{2}\right)(1+\alpha \cos (k x)), x \in\left[0, \frac{2 \pi}{k}\right]$ with periodic conditions in $x$ and $v \in \mathbb{R}$ (cut to $[-10,10]$, assuming that the number of gas particles having a larger absolute velocity is negligible). We take here $k=0.5$ and $\alpha=10^{-2}$.

The hybrid MiMa-Part scheme is compared to MiMa-Grid for different values of $\varepsilon$, from $10^{-2}$ to 1 , in Figure 1. We look at the time evolution of $\log \|E(t)\|_{L^{2}}$ which is known to decrease linearly in time. The kinetic regime ( $\varepsilon=1-$ on the left) is well described but the number of needed particles is quite big: $N=5 \times 10^{5}$. For $\varepsilon=0.5$ (on the right), we note that the boundary layer is captured by both methods. For small values of $\varepsilon$ (for example $10^{-2}$ but decreasing $\varepsilon$ does no more change the curves - on the right), MiMa-Part leads to the diffusion limit, as well as MiMa-Grid. But here, 200 particles are sufficient to represent in a good way the perturbation $g$ and to capture the limit. The cost of MiMa-Part then reduces as $\varepsilon \rightarrow 0$, whereas MiMa-Grid keeps the same complexity.

We then consider the RTE-testcase given by $f(0, x, v)=1+\cos \left(2 \pi\left(x+\frac{1}{2}\right)\right)$, $E=0 \forall t, x \in[0,1]$ with periodic conditions in $x$ and $v \in[-1,1]$.

Results obtained at $t=0.1$ are presented in Figure 2. On the left, MiMa-Part is compared to MiMa-Grid for $\varepsilon=1$ and $\varepsilon=10^{-2}$. In both regimes, our hybrid scheme 

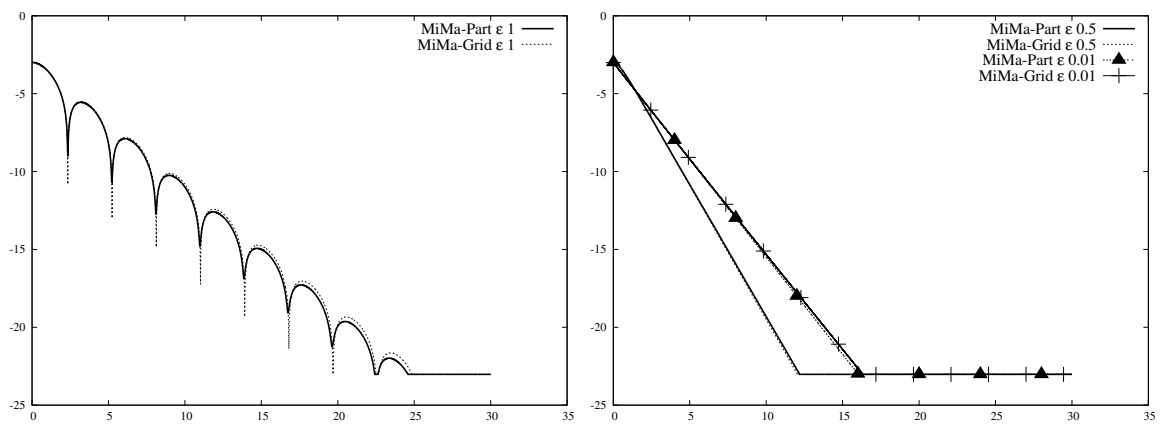

Fig. 1 Landau damping test case. MiMa-Part compared to MiMa-Grid. Electric energy as a function of time $t$ for $\varepsilon=1$ on the left and $\varepsilon=0.5$ and $10^{-2}$ on the right.

gives a good representation of the density $\rho(x)$. These results can also be compared to those of [12]. On the right, we compare MiMa-Part to a full PIC method when $\varepsilon=1$. From the PIC point of view, our hybrid scheme can be seen as a $\delta f$ method (see [4] for example). We thus take the same advantages: the noise due to the probabilistic character of the particles discretization is reduced since it affects only the perturbation $g$, and not the whole function $f$. This noise appears on the representation of $\rho$ when $N$ is too small, and for example in the black curve obtained with Full-PIC and $N=5 \times 10^{4}$. With the same order of $N$, the black line labeled with crosses corresponding to MiMa-Part and $N=4 \times 10^{4}$ is not affected by this noise. Finally, for obtaining a smooth curve with Full-PIC, we have to take $N=5 \times 10^{5}$ (see the dashed line). The cost of the model is directly linked to $N$. To obtained the two smooth curves for $\varepsilon=1$, the computational time is $0.12 s$ for MiMa-Part and $0.47 s$ for Full-PIC.
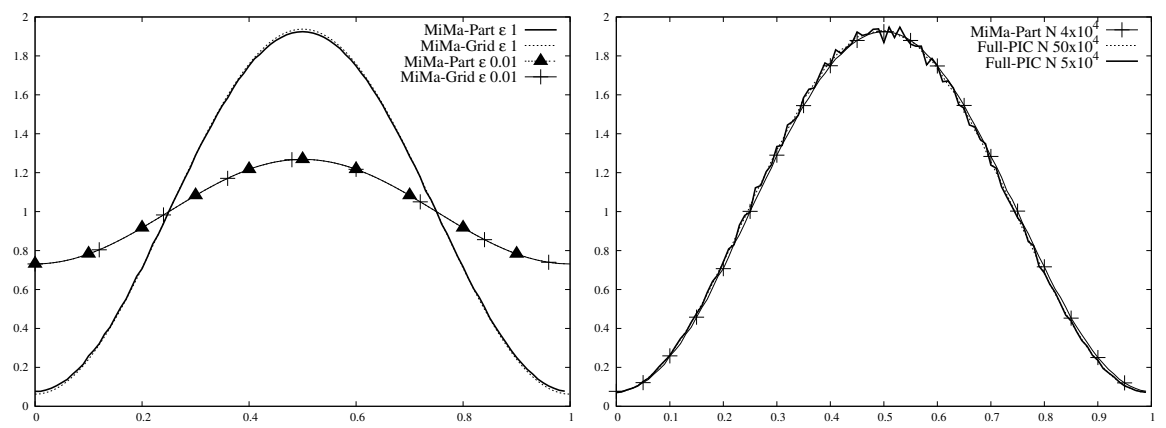

Fig. 2 RTE-testcase. Density $\rho$ as a function of $x$ at time $t=0.1$. MiMa-Part compared to MiMaGrid for $\varepsilon=1$ and $10^{-2}$ on the left and compared to a full PIC method for $\varepsilon=1$ on the right. 


\section{Conclusion and perspectives}

A first extension of the AP hybrid method developed in [5] is presented in this paper, concerning the diffusion scaling. Same conclusions are observed: the scheme is AP and the number of needed particles to represent $g$ in a good way decreases as $\varepsilon \rightarrow 0$. The cost of the hybrid method reduces then at the diffusion limit, whereas it does not depend on $\varepsilon$ in standard phase-space grid methods.

Other possible extensions may be considered and will be the subject of future works. First, it would be interesting to deal with Dirichlet boundary conditions (instead of periodic ones) for enlarging the application field. For the same reason, more general collision operators should be considered, combining this approach with relaxation techniques as in [12]. Extension to higher dimensions of the phase-space is also possible and a comparison with semi-Lagrangian schemes would be interesting.

Acknowledgements. A. Crestetto would like to thank the ANR Project GEONUM (ANR-12-IS01-0004-01), N. Crouseilles the ERC Starting Grant Project GEOPARDI and M. Lemou the ANR Project LODIQUAS (ANR-11-IS01-0003) for their financial supports.

\section{References}

1. Bennoune, M., Lemou, M., Mieussens, L.: Uniformly stable numerical schemes for the Boltzmann equation preserving the compressible Navier-Stokes asymptotics. Journal of Computational Physics 227, 3781-3803 (2008)

2. Berthon, C., Turpault, R.: A numerical correction of the M1-model in the diffusive limit. Discrete and Continuous Dynamical Systems Series S 5, 245-255 (2012)

3. Birdsall, C.K., Langdon, A.B.: Plasma physics via computer simulation. McGraw-Hill New York (1985)

4. Brunner, S., Valeo, E., Krommes, J.A.: Collisional delta-f scheme with evolving background for transport time scale simulations. Physics of Plasmas 6, 4504-4521 (1999)

5. Crestetto, A., Crouseilles, N., Lemou, M.: Kinetic/fluid micro-macro numerical schemes for Vlasov-Poisson-BGK equation using particles. Kinetic and Related Models 5, 787-816 (2012)

6. Crouseilles, N., Lemou, M.: An asymptotic preserving scheme based on a micro-macro decomposition for collisional Vlasov equations: diffusion and high-field scaling limits. Kinetic and Related Models 4, 441-477 (2011)

7. Degond, P., Dimarco, G., Pareschi, L.: The moment guided Monte Carlo method. International Journal for Numerical Methods in Fluids 67, 189-213 (2011)

8. Dimarco, G., Pareschi, L.: Asymptotic preserving implicit-explicit Runge-Kutta methods for non linear kinetic equations. SIAM Journal of Numerical Analysis 51, 1064-1087 (2013)

9. Golse, F., Jin, S., Levermore, C.D.: A domain decomposition analysis for a two-scale linear transport problem. Mathematical Modelling and Numerical Analysis 37, 869-892 (2003)

10. Jin, S.: Efficient asymptotic-preserving (AP) schemes for some multiscale kinetic equations. SIAM Journal on Scientific Computing 21, 441-454 (1999)

11. Klar, A.: An asymptotic-induced scheme for nonstationary transport equations in the diffusive limit. SIAM Journal of Numerical Analysis 35, 1073-1094 (1998)

12. Lemou, M.: Relaxed micro-macro schemes for kinetic equations. Comptes Rendus Mathématique 348, 455-460 (2010)

13. Lemou, M., Mieussens, L.: A new asymptotic preserving scheme based on micro-macro formulation for linear kinetic equations in the diffusion limit. SIAM Journal on Scientific Computing 31, 334-368 (2008) 